\title{
Dual representation of superhedging costs in illiquid markets
}

\author{
Teemu Pennanen*
}

December 21, 2011

\begin{abstract}
This paper studies superhedging of contingent claims in illiquid markets where trading costs may depend nonlinearly on the traded amounts and portfolios may be subject to constraints. We give dual expressions for superhedging costs of financial contracts where claims and premiums are paid possibly at multiple points in time. Besides classical pricing problems, this setup covers various swap and insurance contracts where premiums are paid in sequences. Validity of the dual expressions is proved under new relaxed conditions related to the classical no-arbitrage condition. A new version of the fundamental theorem of asset pricing is given for unconstrained models with nonlinear trading costs.
\end{abstract}

Key words: Illiquidity, superhedging, convex duality

\section{Introduction}

Prices of contingent claims are often expressed in terms of probability measures under which market prices of traded assets are martingales. Traditionally, this is done assuming perfect liquidity and the existence of a cash-account (or a numeraire) which allows for free transfer of positive as well as negative amounts of cash in time; see e.g. $[13,5]$. There is also a growing literature on more realistic market models with transaction costs, portfolio constraints and illiquidity effects; see e.g. Cvitanic and Karatzas [3], Jouini and Kallal [19, 18], Kabanov [22], Schachermayer [42], Çetin, Jarrow and Protter [2], Rokhlin [38, 40], Kaval and Molchanov [24], Dempster, Evstigneev and Taksar [6], Kabanov and Safarian [23], Kreher [25], Pennanen and Penner [32], Pennanen [29, 31, 30] and the references there.

This paper is a continuation of [29, 31], where superhedging of random cashflows was studied in a nonlinear market model with illiquidity effects and portfolio constraints. Our focus is on financial contracts where one sequence of uncertain cash-flows is exchanged for another. Such transactions are common

*Department of Mathematics, King's College London, teemu.pennanen@kcl.ac.uk 
in practice where cash cannot be transferred quite freely in time due to different interest rates and restrictions on borrowing and lending. Going beyond perfect liquidity requires dual elements more general than martingale measures in order to get valid dual representations of hedging costs. In particular, when there is no numeraire, dual variables become sequences that reflect the time-value of money. This happens already in fixed income markets where bond prices are expressed in terms of the zero curve. Moreover, in the presence of nonlinear illiquidity effects, a nonlinear term appears in pricing formulas much like in dual representations of convex risk measures which are not positively homogeneous; see e.g. [13, Section 4.2].

Dual representations of superhedging costs have been obtained for various generalizations of the classical linear model of perfectly liquid markets. Dermody and Rockafellar $[7,8]$ studied superhedging in fixed income markets with

nonlinear trading costs. Jouini and Kallal $[19,18]$ gave dual representations of superhedging costs in market models with bid-ask-spreads and short-sales constraints. More general treatments can be found in Jouini and Napp [20] and Jaschke and Küchler [17] but there too, the employed market models are conical, which excludes nonlinear illiquidity effects. There is also an extensive literature on superhedging of portfolio-valued contingent claims (claims with physical delivery) initiated by Kabanov's [22] introduction of the famous currency market model; see e.g. [42, 23, 32] and their references. The present paper studies claims with cash-delivery in a market model that allows for general convex trading costs and portfolio constraints.

This paper starts by a concise review (Sections 2 and 3) of the approach and main results from $[29,31]$. Section 4 gives a new general condition for the validity of dual representations of superhedging costs. In classical perfectly liquid markets, our conditions coincide with the usual no-arbitrage condition while in unconstrained nonlinear models, the conditions are implied by a generalized no-arbitrage condition analogous to the "robust no-arbitrage" condition introduced by Schachermayer [42] for the currency market model of Kabanov [22]. The new condition allows for a generalization of the "fundamental theorem of asset pricing" to market models with nonlinear trading costs.

\section{Superhedging of claim processes}

Since cash cannot be transferred quite freely in time (due to different interest rates and restrictions on borrowing and lending), it is important to distinguish between payments that occur at different points in time. This is why many financial products in practice provide cash-flows at multiple dates. In a typical transaction, an agent receives a sequence $p=\left(p_{t}\right)_{t=0}^{T}$ of cash-flows in exchange for delivering another $c=\left(c_{t}\right)_{t=0}^{T}$. In the classical setup of mathematical finance, on the other hand, one assumes that positive as well as negative amounts of cash can be transferred through the cash-account (numeraire) so it suffices to consider the case where a single payment at the beginning is exchanged for a random payment at the end. This corresponds to $p=\left(p_{0}, 0, \ldots, 0\right)$ and $c=\left(0, \ldots, 0, c_{T}\right)$. 
Allowing for multiple payout dates, we can also study various swap and insurance contracts which usually involve sequences of payments. We will model the claims $c=\left(c_{t}\right)_{t=0}^{T}$ and premiums $p=\left(p_{t}\right)_{t=0}^{T}$ as adapted sequences in a filtered probability space $\left(\Omega, \mathcal{F},\left(\mathcal{F}_{t}\right)_{t=0}^{T}, P\right)$. That is, $c_{t}$ and $p_{t}$ are $\mathcal{F}_{t}$-measurable random variables, and $\left(\mathcal{F}_{t}\right)_{t=0}^{T}$ is a nondecreasing sequence of sub-sigma-algebras of $\mathcal{F}$ modeling the information available to the trader over time. The space of $\left(\mathcal{F}_{t}\right)_{t=0}^{T}$-adapted sequences of cash flows will be denoted by $\mathcal{M}$.

In many situations, a premium process $p \in \mathcal{M}$ is given and the question is what multiple of $p$ will be sufficient for hedging a claim process $c \in \mathcal{M}$. This is the case e.g. in swap contracts where the critical multiple is known as the swap rate. We define the superhedging cost of a $c \in \mathcal{M}$ as the least multiple of $p$ that allows, given the possibility of dynamic trading, for delivering $c$ without a risk of loosing money. In the special case where $p=(1,0, \ldots, 0)$, this becomes the problem of finding the least amount of initial cash sufficient for superhedging $c$ without subsequent investments.

In order to account for illiquidity effects, we will use the nonlinear market model from $[29,31]$ where a finite set $J$ of assets is traded over finite discretetime $t=0, \ldots, T$. A convex cost process is an $\left(\mathcal{F}_{t}\right)_{t=0}^{T}$-adapted sequence of convex normal integrands on $\mathbb{R}^{J} \times \Omega$ such that $S_{t}(0, \omega)=0$. This means that $S_{t}$ is an extended real-valued function on $\mathbb{R}^{J} \times \Omega$ such that the set-valued mapping $\omega \mapsto$ epi $S_{t}(\cdot, \omega)$ is closed convex-valued and $\mathcal{F}_{t}$-measurable; see e.g. [36, Chapter 14]. The value $S_{t}(x, \omega)$ represents the cost of buying a portfolio $x \in \mathbb{R}^{J}$ at time $t$ and state $\omega$. Convex cost processes extend various market models that have appeared in the literature. The classical model of perfectly liquid markets corresponds to $S_{t}(x, \omega)=s_{t}(\omega) \cdot x$ where $s=\left(s_{t}\right)_{t=0}$ is an $\left(\mathcal{F}_{t}\right)_{t=0}^{T}$-adapted sequence of price vectors. Markets with proportional transaction costs and/or bid-ask-spreads can be modeled with

$$
S_{t}(x, \omega)=\sup \left\{s \cdot x \mid s \in\left[\underline{\mathrm{s}}_{t}(\omega), \bar{s}_{t}(\omega)\right]\right\}
$$

where the $\left(\mathcal{F}_{t}\right)_{t=0}^{T}$-adapted $\mathbb{R}^{J}$-valued processes $\underline{\mathrm{s}}$ and $\bar{s}$ give the bid- and askprices, respectively. Convex cost processes arise naturally also in modern limit order markets, where instantaneous trading costs are nonlinear convex functions of the traded amount; see [26] for a statistical analysis of Copenhagen stock exchange.

In addition to nonlinearities in trading costs, one often faces constraints on the portfolios held. We model constraints by a sequence $D=\left(D_{t}\right)_{t=0}^{T}$ of setvalued mappings from $\Omega$ to $\mathbb{R}^{J}$ such that each $D_{t}$ is closed convex-valued and $\mathcal{F}_{t}$-measurable in the sense that

$$
\left\{\omega \in \Omega \mid D_{t}(\omega) \cap U \neq \emptyset\right\} \in \mathcal{F}_{t}
$$

for every open $U \subset \mathbb{R}^{J}$. We assume that $0 \in D_{t}$ almost surely, i.e. that the zero portfolio is always feasible. The classical unconstrained model corresponds to $D \equiv \mathbb{R}^{J}$. Short sale constraints, studied e.g. in Cvitanic and Karatzas [3] and Jouini and Kallal [18] corresponds to $D \equiv \mathbb{R}_{+}^{J}$. 
The portfolio held over period $(t, t+1]$ will be denoted by $x_{t}$. Dynamic trading strategies are described by $\left(\mathcal{F}_{t}\right)_{t=0}^{T}$-adapted portfolio processes $x=\left(x_{t}\right)_{t=0}^{T}$. The linear space of adapted portfolio strategies will be denoted by $\mathcal{N}$. The superhedging problem can now be written as

$$
\begin{array}{lc}
\text { minimize } & \alpha \text { over } \quad \alpha \in \mathbb{R}, x \in \mathcal{N}_{0} \\
\text { subject to } & S_{t}\left(\Delta x_{t}\right)+c_{t} \leq \alpha p_{t}, x_{t} \in D_{t}, t=0, \ldots, T,
\end{array}
$$

where $\mathcal{N}_{0}:=\left\{x \in \mathcal{N} \mid x_{T}=0\right\}, \Delta x_{t}:=x_{t}-x_{t-1}$ and all constraints are required to hold almost surely. Here and in what follows, we always define $x_{-1}:=0$. The elements of $\mathcal{N}_{0}$ thus represent portfolio processes that start and end at liquidated positions. The optimal value, which will be denoted by $\pi(c)$, gives the least multiple of the premium $p$ we would need in order to deliver the claim $c$ without the risk of a positive cost at any point in time.

Example 1 (Liquid markets) Assume that there is a perfectly liquid asset (numeraire), say $0 \in J$, such that

$$
\begin{aligned}
S_{t}(x, \omega) & =x^{0}+\tilde{S}_{t}(\tilde{x}, \omega), \\
D_{t}(\omega) & =\mathbb{R} \times \tilde{D}_{t}(\omega),
\end{aligned}
$$

where $x=\left(x^{0}, \tilde{x}\right)$ and $\tilde{S}$ and $\tilde{D}$ are the cost process and the constraints for the remaining risky assets $\tilde{J}=J \backslash\{0\}$. In this case, we can use the budget constraint to substitute out the numeraire from problem (1). Indeed, defining

$$
x_{t}^{0}=x_{t-1}^{0}-\tilde{S}_{t}\left(\Delta \tilde{x}_{t}\right)-c_{t}+\alpha p_{t} \quad t=0, \ldots, T-1,
$$

the budget constraint holds as an equality for $t=1, \ldots, T-1$ and

$$
x_{T-1}^{0}=-\sum_{t=0}^{T-1} \tilde{S}_{t}\left(\Delta \tilde{x}_{t}\right)-\sum_{t=0}^{T-1} c_{t}+\alpha \sum_{t=0}^{T-1} p_{t} .
$$

Problem (1) can then be written as

$$
\begin{array}{cc}
\text { minimize } & \alpha \quad \text { over } \quad \alpha \in \mathbb{R}, x \in \mathcal{N}_{0} \\
\text { subject to } & \sum_{t=0}^{T} S_{t}\left(\Delta \tilde{x}_{t}\right)+\sum_{t=0}^{T} c_{t} \leq \alpha \sum_{t=0}^{T} p_{t}, \\
& \tilde{x}_{t} \in \tilde{D}_{t}, t=0, \ldots, T .
\end{array}
$$

Thus, in the presence of a numeraire, the timing of payments is irrelevant; only the cumulated claims matter. If the cost process $\tilde{S}$ is linear with $\tilde{S}_{t}(\tilde{x}, \omega)=$ $\tilde{s}_{t}(\omega) \cdot \tilde{x}$, the cumulated trading costs can be written as the stochastic integral

$$
\sum_{t=0}^{T} \tilde{S}_{t}\left(\Delta \tilde{x}_{t}\right)=\sum_{t=0}^{T} \tilde{s}_{t} \cdot \Delta \tilde{x}_{t}=-\sum_{t=0}^{T-1} \tilde{x}_{t} \cdot \Delta \tilde{s}_{t+1} .
$$


In particular, if $p=(1,0, \ldots, 0)$, we can then write problem (2) as

$$
\begin{array}{cc}
\text { minimize } & \alpha \quad \text { over } \quad \alpha \in \mathbb{R}, x \in \mathcal{N}_{0} \\
\text { subject to } & \sum_{t=0}^{T} c_{t} \leq \alpha+\sum_{t=0}^{T-1} \tilde{x}_{t} \cdot \Delta \tilde{s}_{t+1}, \\
& \tilde{x}_{t} \in \tilde{D}_{t}, t=0, \ldots, T .
\end{array}
$$

In the special case $D_{t} \equiv \mathbb{R}^{J}$, this becomes the classical superhedging problem; see [13, 5, 23] and their references. The above corresponds to the constrained extensions studied in Jouini and Kallal [18] and Föllmer and Schied [12].

The superhedging cost can be expressed concisely as

$$
\pi(c)=\inf \{\alpha \in \mathbb{R} \mid c-\alpha p \in \mathcal{C}\},
$$

where

$$
\mathcal{C}=\left\{c \in \mathcal{M} \mid \exists x \in \mathcal{N}_{0}: S_{t}\left(\Delta x_{t}\right)+c_{t} \leq 0, x_{t} \in D_{t}\right\}
$$

is the set of claim processes that can be superhedged at zero cost. It is easily checked that $\mathcal{C}$ is convex. It follows that the superhedging cost $\pi$ is a convex function on $\mathcal{M}$; see [31, Proposition 4.1]. If $\mathcal{C}$ is a cone, which is the case e.g. in the classical liquid market model and in models with proportional transaction costs, $\pi$ is positively homogeneous. This corresponds to "constant returns to scale" in superhedging of claim processes. In the presence of nonlinear illiquidity effects, positive homogeneity is lost in general.

The convexity of $\pi$ greatly facilitates the analysis of superhedging costs. In particular, dual representations for $\pi$ can be derived from the classical biconjugate theorem as soon as $\pi$ is proper (not identically $+\infty$ and never taking the value $-\infty$ ) and lower semicontinuous with respect to an appropriate topology. This is the strategy we will follow in this paper. Proposition 2 below gives sufficient conditions for properness and lower semicontinuity of $\pi$.

The space $\mathcal{M}$ of $\left(\mathcal{F}_{t}\right)_{t=0^{T}}^{T}$-adapted claim processes is a subspace of the space $L^{0}\left(\Omega, \mathcal{F}, P ; \mathbb{R}^{T+1}\right)$ of (equivalence classes of ) $\mathbb{R}^{T+1}$-valued measurable functions. We can thus equip $\mathcal{M}$ with the topology of convergence in probability (in measure). This topology is convenient since it is weaker than the topologies used in the duality theory below. The following is from [29]. We give here a somewhat simplified proof.

Proposition 2 Assume that $\mathcal{C}$ is closed in probability. Then $\pi(c)>-\infty$ for every $c \in \mathcal{M}$ if and only if $\pi(0)>-\infty$ in which case $\pi$ is lower semicontinuous with respect to convergence in probability and the infimum in $\pi(c)=\inf \{\alpha \mid c-$ $\alpha p \in \mathcal{C}\}$ is attained for every $c \in \operatorname{dom} \pi$.

Proof. When $\mathcal{C}$ is closed in probability, the set $\left\{\alpha \in \mathbb{R} \mid c+\alpha c^{\prime} \in \mathcal{C}\right\}$ is closed in $\mathbb{R}$ for every $c, c^{\prime} \in \mathcal{M}$. Indeed, if $c+\alpha^{\nu} c^{\prime} \in \mathcal{C}$ and $\alpha^{\nu} \rightarrow \alpha$, then

$$
P\left(\left|c+\alpha^{\nu} c^{\prime}-c-\alpha c^{\prime}\right| \geq \varepsilon\right)=P\left(\left|\alpha^{\nu}-\alpha\right|\left|c^{\prime}\right| \geq \varepsilon\right) \rightarrow 0
$$


for every $\varepsilon>0$. The above means that $c+\alpha^{\nu} c^{\prime}$ converges to $c+\alpha c^{\prime}$ in probability, so the closedness of $\mathcal{C}$ implies $c+\alpha c^{\prime} \in \mathcal{C}$. The first claim and the attainment of the infimum thus follow from [31, Proposition 4.2], which is a simple exercise in convex analysis.

Let $c^{\nu} \rightarrow c$ in probability such that $\pi\left(c^{\nu}\right) \leq \gamma$. Since the infimum is attained, there exist $\alpha^{\nu} \leq \gamma$ such that $c^{\nu}-\alpha^{\nu} p \in \mathcal{C}$. If $\left(\alpha^{\nu}\right)$ clusters at some $\alpha$, then $c-\alpha p \in \mathcal{C}$, by the closedness of $\mathcal{C}$, so that $\pi(c) \leq \gamma$. It thus suffices to show that the sequence $\left(\alpha^{\nu}\right)$ is bounded from below. If it is not, we may assume, by passing to a subsequence if necessary, that $\left(\alpha^{\nu}\right)$ decreases monotonically to $-\infty$. Given $\beta>0$, we have

$$
\frac{1}{-\alpha^{\nu}} c^{\nu}+p \in \frac{1}{-\alpha^{\nu}} \mathcal{C} \subseteq \beta \mathcal{C}
$$

for $\nu$ large enough. Indeed, since $\mathcal{C}$ is a convex set containing the origin, we have $\beta_{1} \mathcal{C} \subseteq \beta_{2} \mathcal{C}$ whenever $0<\beta_{1} \leq \beta_{2}$. Since $\left(c^{\nu}\right)$ converges and $\mathcal{C}$ is closed, we thus get $p \in \beta \mathcal{C}$. Since $\beta>0$ was arbitrary, this implies $\pi(0)=-\infty$ in contradiction to our assumption.

Closedness of the set $\mathcal{C}$ assumed in Proposition 3 will be essential also in the duality theory in Section 3. Sufficient conditions for the closedness will be given in Section 4 where it is shown, in particular, that it holds in classical liquid market models that are free of "arbitrage".

The condition $\pi(0)>-\infty$ is a minimal compatibility condition on the market model $(S, D)$ (which defines $\mathcal{C}$ ) and the premium process $p \in \mathcal{M}$. If $\pi(0)=-\infty$, we could superhedge arbitrary multiples of $p$ by costless trading in the market. It would not make sense to ask for such premiums as a compensation for delivering another claim $c \in \mathcal{M}$. If $\mathcal{C}$ is a cone, as happens when $S$ is sublinear (i.e. convex and positively homogeneous) and $D$ is conical, we have $\pi(0)>-\infty$ if and only if $\pi(0) \geq 0$. If, in addition, $p=(1,0, \ldots, 0)$, the condition $\pi(0) \geq 0$ means that it is not possible to superhedge the zero claim when starting with strictly negative initial wealth. In the terminology of Ingersoll [15], this means that there is no "arbitrage of the second kind" or in the terminology of Dermody and Rockafellar $[7,8]$, that the market satisfies the "weak no-arbitrage" condition.

\section{Dual representation of the superhedging cost}

This section gives dual representations of the superhedging cost $\pi$ on the space $\mathcal{M}^{1}:=\mathcal{M} \cap L^{1}\left(\Omega, \mathcal{F}, P ; \mathbb{R}^{T+1}\right)$ of integrable claim processes. We generalize some classical results from the theory of linear models of perfectly liquid markets to the nonlinear model described above. This illustrates, in particular, how some well-known risk neutral pricing formulas are affected by illiquidity. The dual representations may also be useful in certain numerical techniques for computing lower bounds on the superhedging cost. In the case of complete markets, good results have been obtained in pricing of American options in [37] and [14]. A more general approach is outlined in [30, Remark 3.1]. 
The bilinear form

$$
\langle c, y\rangle:=E \sum_{t=0}^{T} c_{t} y_{t}
$$

puts $\mathcal{M}^{1}$ and the space $\mathcal{M}^{\infty}:=\mathcal{M} \cap L^{\infty}\left(\Omega, \mathcal{F}, P ; \mathbb{R}^{T+1}\right)$ of essentially bounded processes in separating duality. This pairing gives a sufficient structure for using classical duality methods from convex analysis; see e.g. [35, 9]. The elements of $\mathcal{M}^{\infty}$ represent deflators (or shadow prices, state price densities, stochastic term structures, ...) that generalize martingale measures often used in the classical perfectly liquid setup; see Example 6 below. When there is no numeraire, more general dual objects from $\mathcal{M}^{\infty}$ are needed in order to bring out the term structure of cash flows. In a deterministic setting, the dual variables $y \in \mathcal{M}^{\infty}$ represent the term structure of interest rates (more precisely, the "zero curve"); see also $[7,8]$.

The dual representation of $\pi$ will be given in terms of the support function

$$
\sigma_{\mathcal{C}^{1}}(y):=\sup _{c \in \mathcal{C}^{1}}\langle c, y\rangle
$$

of the set $\mathcal{C}^{1}:=\mathcal{C} \cap \mathcal{M}^{1}$ of integrable claim processes that can be superhedged at zero cost. The support function is a lower semicontinuous sublinear function on $\mathcal{M}^{\infty}$. In the terminology of microeconomic analysis, $\sigma_{\mathcal{C}^{1}}$ is the profit function associated with the production set $\mathcal{C}^{1}$; see e.g. [1] or [27]. It gives the maximum amount of wealth one could produce by trading in financial markets in a world where the cash-flows $c \in \mathcal{C}^{1}$ are worth $\langle c, y\rangle$. The following is from [31]. We include the simple proof for completeness.

Proposition 3 Assume that $\mathcal{C}$ is closed in probability and let $p \in \mathcal{M}^{1}$. We have $\pi(0)>-\infty$ if and only if there is a $y \in \operatorname{dom} \sigma_{\mathcal{C}^{1}}$ such that $\langle p, y\rangle=1$. In that case,

$$
\pi(c)=\sup _{y \in \mathcal{M}^{\infty}}\left\{\langle c, y\rangle-\sigma_{\mathcal{C}^{1}}(y) \mid\langle p, y\rangle=1\right\}
$$

on $\mathcal{M}^{1}$.

Proof. When $p \in \mathcal{M}^{1}$, the restriction $\bar{\pi}$ of $\pi$ to $\mathcal{M}^{1}$ can be written as $\bar{\pi}(c)=$ $\inf \left\{\alpha \mid c-\alpha p \in \mathcal{C}^{1}\right\}$. Its conjugate $\bar{\pi}^{*}: \mathcal{M}^{\infty} \rightarrow \overline{\mathbb{R}}$ can be expressed as

$$
\begin{aligned}
\bar{\pi}^{*}(y) & =\sup _{c \in \mathcal{M}^{1}}\{\langle c, y\rangle-\pi(c)\} \\
& =\sup _{c \in \mathcal{M}^{1}, \alpha \in \mathbb{R}}\left\{\langle c, y\rangle-\alpha \mid c-\alpha p \in \mathcal{C}^{1}\right\} \\
& =\sup _{c^{\prime} \in \mathcal{M}^{1}, \alpha \in \mathbb{R}}\left\{\left\langle c^{\prime}+\alpha p, y\right\rangle-\alpha \mid c^{\prime} \in \mathcal{C}^{1}\right\} \\
& =\sup _{c^{\prime} \in \mathcal{M}^{1}, \alpha \in \mathbb{R}}\left\{\left\langle c^{\prime} y\right\rangle+\alpha[\langle p, y\rangle-1] \mid c^{\prime} \in \mathcal{C}^{1}\right\} \\
& = \begin{cases}\sigma_{\mathcal{C}^{1}}(y) & \text { if }\langle p, y\rangle=1, \\
+\infty & \text { otherwise. }\end{cases}
\end{aligned}
$$


If $\pi(0)=-\infty$, then $\bar{\pi}^{*} \equiv+\infty$. On the other hand, if $\pi(0)>-\infty$, then by Proposition 2, $\pi$ is proper and lsc on $\mathcal{M}$ with respect to convergence in measure, which in turn implies that $\bar{\pi}$ is proper and lsc on $\mathcal{M}^{1}$ with respect to the $L^{1}$-norm topology. Since the norm topology is compatible with the pairing of $\mathcal{M}^{1}$ and $\mathcal{M}^{\infty}$, the rest now follows from the classical biconjugate theorem; see e.g. [35, Theorem 5].

The dual representation in Proposition 3 may be interpreted as follows. If all random cash-flows $c \in \mathcal{M}$ are valued by $\langle c, y\rangle$, then $\langle c, y\rangle-\sigma_{\mathcal{C}^{1}}(y)$ gives the net cost of delivering $c$ while trading optimally in financial markets. The superhedging cost of $c$ is obtained by taking the supremum of $\langle c, y\rangle-\sigma_{\mathcal{C}^{1}}(y)$ over all deflators $y \in \mathcal{M}^{\infty}$ that price the premium process $p$ at one. When $\mathcal{C}^{1}$ is a cone, we have $\sigma_{\mathcal{C}^{1}}=\delta_{\mathcal{C}^{*}}$, where

$$
\mathcal{C}^{*}=\left\{y \in \mathcal{M}^{\infty} \mid\langle c, y\rangle \leq 0 \forall c \in \mathcal{C}^{1}\right\}
$$

is the polar cone of $\mathcal{C}^{1}$. Here $\delta_{\mathcal{C}^{*}}$ denotes the indicator function of $\mathcal{C}^{*}$ defined as $\delta_{\mathcal{C}^{*}}(y)=0$ for $y \in \mathcal{C}^{*}$ and $\delta_{\mathcal{C}^{*}}(y)=+\infty$ otherwise. In the conical case, the dual representation in Proposition 3 can thus be written as

$$
\pi(c)=\sup _{y \in \mathcal{C}^{*}}\{\langle c, y\rangle \mid\langle p, y\rangle=1\} .
$$

It turns out (see Example 6 below) that in the case of the classical linear market model, the deflators $y$ in the above formula are precisely the martingale density processes for the underlying price process.

A reader familiar with convex risk measures has probably noticed the similarity between the above expressions and the dual representations of convex risk measures. The support function $\sigma_{\mathcal{C}^{1}}$ corresponds to the penalty function in the dual representation of a convex risk measure; see e.g. [13, Section 4.2]. The penalty function of a sublinear risk measure reduces to an indicator function just like the the support function of a conical production set above; see [13, Corollary 4.18].

In order to derive more concrete expressions for the support function of $\mathcal{C}^{1}$, we will assume that the cost process $S$ is integrable in the sense that $S_{t}(x, \cdot)$ is integrable for every $x \in \mathbb{R}^{J}$ and $t=0, \ldots, T$. A linear cost process $S_{t}(x, \omega)=$ $s_{t}(\omega) \cdot x$, for example, is integrable if and only if $s_{t}$ have integrable components. The following is from [29, Lemma A.1].

Lemma 4 If $S$ is integrable, then

$$
\sigma_{\mathcal{C}^{1}}(y)=\inf _{v \in \mathcal{N}^{1}}\left\{\sum_{t=0}^{T} E\left(y_{t} S_{t}\right)^{*}\left(v_{t}\right)+\sum_{t=0}^{T-1} E \sigma_{D_{t}}\left(E_{t}\left[\Delta v_{t+1}\right]\right)\right\}
$$

for every $y \in \mathcal{M}_{+}^{\infty}$ while $\sigma_{C^{1}}(y)=+\infty$ for $y \notin \mathcal{M}_{+}^{\infty}$. Moreover, the infimum is attained for every $y \in \mathcal{M}_{+}^{\infty}$. 
Here $\mathcal{N}^{1}$ denotes the space of integrable $\left(\mathcal{F}_{t}\right)_{t=0^{T}}^{T}$-adapted $\mathbb{R}^{J}$-valued processes, $E_{t}$ stands for the conditional expectation with respect to $\mathcal{F}_{t}$ while $\left(y_{t} S_{t}\right)^{*}$ and $\sigma_{D_{t}}$ are the $\mathcal{F}_{t}$-measurable normal integrands defined by

$$
\left(y_{t} S_{t}\right)^{*}(v, \omega):=\sup _{x \in \mathbb{R}^{J}}\left\{x \cdot v-y_{t}(\omega) S_{t}(x, \omega)\right\}
$$

and

$$
\sigma_{D_{t}}(v, \omega):=\sup _{x \in D_{t}(\omega)} x \cdot v .
$$

Since $S_{t}(0, \omega)=0$ and $0 \in D_{t}(\omega)$, these are nonnegative convex functions.

When $S$ is sublinear and $D$ is conical, the set $\mathcal{C}$ is a cone with polar $\mathcal{C}^{*}=$ $\left\{y \in \mathcal{M}^{\infty} \mid \sigma_{\mathcal{C}^{1}}(y) \leq 0\right\}$. Lemma 4 then gives

$$
\mathcal{C}^{*}=\left\{y \in \mathcal{M}_{+}^{\infty} \mid \exists v \in \mathcal{N}^{1}:\left(y_{t} S_{t}\right)^{*}\left(v_{t}\right) \leq 0, E_{t}\left[\Delta v_{t+1}\right] \in D_{t}^{*}\right\} .
$$

The condition $\left(y_{t} S_{t}\right)^{*}\left(v_{t}\right) \leq 0$ means that $y_{t}(\omega) S_{t}(x, \omega) \geq x \cdot v_{t}(\omega)$ for all $x \in \mathbb{R}^{J}$, or equivalently, that $v_{t}(\omega) \in y(\omega)_{t} \partial S_{t}(0, \omega)$, where $\partial S_{t}(x, \omega)$ is the set of vectors $v \in \mathbb{R}^{J}$ defined by

$$
S_{t}(x, \omega) \geq S_{t}(0, \omega)+v \cdot x \quad \forall x \in \mathbb{R}^{J} .
$$

In the terminology of convex analysis, $\partial S_{t}(0, \omega)$ is the subdifferential of $S_{t}(\cdot, \omega)$ at 0 and its elements are called subgradients. In the case of limit order markets, $S_{t}(0, \omega)$ gives the set of price vectors bounded by bid- and ask-prices; see [26]. In the conical case, we thus have

$$
\mathcal{C}^{*}=\left\{y \in \mathcal{M}_{+}^{\infty} \mid \exists v \in \mathcal{N}^{1}: v_{t} \in y_{t} \partial S_{t}(0), E_{t}\left[\Delta v_{t+1}\right] \in D_{t}^{*}\right\} .
$$

This can be rewritten as follows.

Corollary 5 If $S$ is sublinear and integrable and if $D$ is conical, then

$$
\mathcal{C}^{*}=\left\{y \in \mathcal{M}_{+}^{\infty} \mid \exists s \in \mathcal{N}: y s \in \mathcal{N}^{1}, s_{t} \in \partial S_{t}(0), E_{t}\left[\Delta\left(y_{t+1} s_{t+1}\right)\right] \in D_{t}^{*}\right\} .
$$

Example 6 (Cash-account) When $S_{t}(x, \omega)=x^{0}+\tilde{S}_{t}(\tilde{x}, \omega)$ and $D_{t}(\omega)=$ $\mathbb{R} \times \tilde{D}_{t}(\omega)$ as in Example 1, we get

$$
\partial S_{t}(x, \omega)=\{1\} \times \partial \tilde{S}_{t}(\tilde{x}, \omega) \text { and } D_{t}^{*}(\omega)=\{0\} \times \tilde{D}_{t}(\omega)^{*},
$$

so the elements of $\mathcal{C}^{*}$ are nonnegative martingales $y$ for which there exists an adapted process $\tilde{s}$ evolving in $\partial \tilde{S}_{t}(0, \omega)$ such that $E_{t}\left[\Delta\left(y_{t+1} s_{t+1}\right)\right] \in \tilde{D}_{t}^{*}(\omega)$. The elements $y \in \mathcal{C}^{*}$ with $y_{0}=1$ are thus density processes of measures $Q$ which are absolutely continuous with respect to $P$. For such a $y$, we can write the condition $E_{t}\left[\Delta\left(y_{t+1} s_{t+1}\right)\right] \in \tilde{D}_{t}^{*}$ as $E_{t}^{Q}\left[\Delta s_{t+1}\right] \in \tilde{D}_{t}^{*}$ (use [13, Proposition A.12] and the fact that $D_{t}^{*}$ is a cone). Choosing $p=(1,0, \ldots, 0)$ in Proposition 3, we thus get the dual representation

$$
\pi(c)=\sup _{Q \in \mathcal{P}} E^{Q} \sum_{t=0}^{T} c_{t},
$$


where $\mathcal{P}$ is the set of probability measures $Q \ll P$ for which there is an adapted process $\tilde{s}$ evolving in $\partial \tilde{S}_{t}(0, \omega)$ such that $E_{t}^{Q}\left[\Delta \tilde{s}_{t}\right] \in \tilde{D}_{t}^{*}(\omega)$. In the unconstrained case where $D \equiv \mathbb{R}^{J}$, we have $\tilde{D}_{t}^{*}(\omega) \equiv\{0\}$ so $\mathcal{P}$ becomes the set of probability measures under which some process in $\partial \tilde{S}(0, \omega)$ is a martingale. Such a pricing formula was obtained in Jouini and Kallal [19] for models with bid-ask-spreads. In the linear case where $S(x, \omega)=x^{0}+\tilde{s}_{t}(\omega) \cdot \tilde{x}$, the set $\mathcal{P}$ is the set of absolutely continuous martingale measures for $\tilde{s}$ and the above formula reduces to the wellknown expression for the superhedging cost in classical perfectly liquid market models; see e.g. [5, Section 2.4], [13, Section 5.3], [23, Chapter 2] and the references there.

The above example shows that, in models with a cash-account (a numeraire), superhedging costs can be expressed in terms of probability (martingale) measures. Without a cash-account, however, a richer set of dual elements is needed for dual representations.

Example 7 (Short-sales constraints) When $S_{t}(x, \omega)=s_{t}(\omega) \cdot x$ and $D$ is conical, we get

$$
\mathcal{C}^{*}=\left\{y \in \mathcal{M}_{+}^{\infty} \mid y s \in \mathcal{M}^{1}, E_{t}\left[\Delta\left(y_{t+1} s_{t+1}\right)\right] \in D_{t}^{*}\right\} .
$$

When $D_{t}(\omega)=\mathbb{R}_{+}^{J}$, we have $D_{t}^{*}(\omega) \equiv \mathbb{R}_{-}^{J}$, so the set $\mathcal{C}^{*}$ consist of processes $y \in \mathcal{M}^{\infty}$ such that ys is a supermartingale. If one of the assets has strictly positive constant unit price, then $y$ is itself a supermartingale so it has the Itô-Watanabe decomposition $y=M A$ where $M$ is a martingale and $A$ is a nonincreasing predictable process with values in [0,1]; see Fölmer [11]. We can then write the dual representation in Proposition 3 for $p=(1,0, \ldots, 0)$ as

$$
\pi(c)=\sup \left\{E^{Q} \sum_{t=0}^{T} c_{t} A_{t} \mid(Q, A) \in \mathcal{Q} \mathcal{A}\right\},
$$

where $\mathcal{Q} \mathcal{A}$ is the set of pairs of probability measures $Q$ and predictable nonincreasing processes $A \geq 0$ with $A_{0}=1$ such that $A s$ is a supermartingale under Q. This is a discrete-time version of Jouini and Kallal [18, Theorem 2.1(iii)]. Actually, the model of [18] allows for bid-ask-spreads and constraints of the form $x_{t}^{j} \leq 0$ for some assets (which is convenient for modeling different interest rates for lending and borrowing). Such extensions are easily treated with Corollary 5. More general portfolio constraints have been studied in Napp [28], Evstigneev, Schürger and Taksar [10] and Rokhlin [39].

It is worth emphasizing that we have not assumed the absence of arbitrage in the market model. By Proposition 3, the dual representations of the superhedging cost are valid as soon as the set $\mathcal{C}$ is closed and $\pi(0)>-\infty$. In the classical linear model of perfectly liquid markets, both conditions are implied by the no-arbitrage condition; see Example 9 below and the discussion at the end of Section 2. 
Under the no-arbitrage condition, the set $\mathcal{P}$ of absolutely continuous martingale measures in the linear case of Example 6 can be replaced by martingale measures that are equivalent to the original measure $P$. Indeed, the no-arbitrage condition implies (see Example 15 below) that there is a $P^{e} \in \mathcal{P}$ which is equivalent to $P$ and then, for any $P^{a} \in \mathcal{P}$ and $\alpha \in(0,1]$ the measure $\alpha P^{e}+(1-\alpha) P^{a}$ is also an equivalent martingale measure.

\section{Closedness of $\mathcal{C}$ and no-arbitrage criteria}

The dual representations derived above depend on the closedness of the set $\mathcal{C}$ of claim processes that can be superhedged without a cost in a market described by a cost process $S=\left(S_{t}\right)_{t=0}^{T}$ and constraints $D=\left(D_{t}\right)_{t=0}^{T}$. This is a highly nontrivial condition whose proof already in the linear case requires sophisticated techniques from measure theory; see Schachermayer [41, Section 2]. The present section gives sufficient conditions for the closedness of $\mathcal{C}$ in the general convex case by applying the abstract closedness result from [30, Section 5]. While the no-arbitrage criterion is not necessary for the validity of dual representations of the superhedging cost, it turns out that the closedness of $\mathcal{C}$, which we do need, often follows from the no-arbitrage condition or appropriate generalizations of it.

Given a market model $(S, D)$, we obtain another market model $\left(S^{\infty}, D^{\infty}\right)$ by defining

$$
\begin{aligned}
S_{t}^{\infty}(x, \omega) & =\sup _{\alpha>0} \frac{S_{t}(\alpha x, \omega)}{\alpha}, \\
D_{t}^{\infty}(\omega) & =\bigcap_{\alpha>0} \alpha D_{t}(\omega) .
\end{aligned}
$$

Indeed, the required measurability properties hold by [36, Exercises 14.54 and 14.21] while the convexity and topological properties come directly from the definitions. Moreover, the functions $S_{t}^{\infty}(\cdot, \omega)$ are positively homogeneous and the sets $D_{t}^{\infty}(\omega)$ are cones. We have $(S, D)=\left(S^{\infty}, D^{\infty}\right)$ if and only if $S$ is sublinear and $D$ is conical. The set

$$
\mathcal{L}=\left\{x \in \mathcal{N}_{0} \mid S_{t}^{\infty}\left(\Delta x_{t}\right) \leq 0, x_{t} \in D_{t}^{\infty}\right\}
$$

is a convex cone in $\mathcal{N}_{0}$.

Theorem 8 If $\mathcal{L}$ is linear, $\mathcal{C}$ is closed.

Proof. We have $\mathcal{C}=\{c \in \mathcal{M} \mid \exists x \in \mathcal{N}:(x(\omega), c(\omega)) \in C(\omega) P$-a.s. $\}$, where

$$
C(\omega)=\left\{(x, u) \in \mathbb{R}^{n} \times \mathbb{R}^{m} \mid S_{t}\left(\Delta x_{t}, \omega\right)+u_{t} \leq 0, x_{t} \in D_{t}(\omega) t=0, \ldots, T\right\} .
$$

with $n=(T+1)|J|, m=T+1$ and $D_{T}:=\{0\}$. By [30, Theorem 5.2] $]^{1}, \mathcal{C}$ is closed provided

$$
\left\{x \in \mathcal{N} \mid(x(\omega), 0) \in C^{\infty}(\omega) P \text {-a.s. }\right\}
$$

\footnotetext{
${ }^{1}$ The statement of [30, Theorem 5.2] gives the closedness of $\mathcal{C}^{1}$ in the weak topology $\sigma\left(L^{1}, L^{\infty}\right)$, but the proof of [30, Theorem 5.2] also gives the closedness of $\mathcal{C}$ in probability.
} 
is a linear space. Here $C^{\infty}(\omega):=\bigcap_{\alpha>0} \alpha C(\omega)$. By [34, Corollary 8.3.3 and Theorem 8.7],

$$
C(\omega)^{\infty}=\left\{(x, u) \mid S_{t}^{\infty}\left(\Delta x_{t}, \omega\right)+u_{t} \leq 0, x_{t} \in D_{t}^{\infty}(\omega)\right\},
$$

which completes the proof.

The abstract linearity condition in Theorem 8 can be seen as a generalization of the no-arbitrage condition in the case of perfectly liquid markets. A market model is said to allow for arbitrage if there is a self-financing trading strategy that superhedges a nontrivial nonnegative claim. In the present model, this would mean that there is an $x \in \mathcal{N}_{0}$ with $x_{t} \in D_{t}$ and $S_{t}\left(\Delta x_{t}\right) \leq 0$ almost surely for every $t$ and $S_{t}\left(\Delta x_{t}\right)<0$ with positive probability for some $t$. The absence of arbitrage means that

$$
\mathcal{C} \cap \mathcal{M}_{+}=\{0\},
$$

where $\mathcal{M}_{+}:=\left\{c \in \mathcal{M} \mid c_{t} \geq 0 P\right.$-a.s. $\left.\forall t\right\}$. If there are no portfolio constraints and if one of the assets has unit price equal to one (see Examples 1 and 6), we can write the no-arbitrage condition as

$$
\mathcal{C}_{T} \cap L_{+}^{0}=\{0\},
$$

where

$$
\mathcal{C}_{T}=\left\{c \in L^{0}\left(\Omega, \mathcal{F}_{T}, P\right) \mid \exists x \in \mathcal{N}_{0}: c \leq \sum_{t=0}^{T-1} x_{t} \cdot \Delta s_{t+1}\right\} .
$$

This is the traditional formulation of the no-arbitrage condition in mathematical finance; see e.g. $[4,13,5]$.

Example 9 (Liquid markets) When $S_{t}(x, \omega)=s_{t}(\omega) \cdot x$ and $D_{t} \equiv \mathbb{R}^{J}$,

$$
\mathcal{L}=\left\{x \in \mathcal{N}_{0} \mid s_{t} \cdot \Delta x_{t} \leq 0\right\},
$$

so the linearity of $\mathcal{L}$ means that any self-financing trading strategy $x \in \mathcal{N}_{0}$ satisfies $s_{t} \cdot \Delta x_{t}=0$ almost surely for all $t$. This is exactly the no-arbitrage condition.

In general nonlinear models, the no-arbitrage condition (NA) is neither sufficient nor necessary for closedness of $\mathcal{C}$; see [31, Section 6] for examples. Nevertheless, the situation in Example 9 can be generalized as shown by Schachermayer [42] in the setting of the currency market model of Kabanov [22]. In the present model, Example 9 can be generalized as follows.

We will say that a cost process $S=\left(S_{t}\right)_{t=0}^{T}$ satisfies the robust no-arbitrage (RNA) condition if there exists an unconstrained arbitrage-free market model with sublinear $\tilde{S}=\left(\tilde{S}_{t}\right)_{t=0}^{T}$ such that

$$
\begin{array}{ll}
\tilde{S}_{t}(x, \omega) \leq S_{t}^{\infty}(x, \omega) & \forall x \in \mathbb{R}^{J}, \\
\tilde{S}_{t}(x, \omega)<S_{t}^{\infty}(x, \omega) & \forall x \notin \operatorname{lin} S_{t}(\cdot, \omega) .
\end{array}
$$


Here $\operatorname{lin} S_{t}(\cdot, \omega):=\left\{x \in \mathbb{R}^{J} \mid S_{t}^{\infty}(-x, \omega) \leq-S_{t}^{\infty}(x, \omega)\right\}$ is the so called lineality space of $S_{t}(\cdot, \omega)$; see [34, page 70]. The lineality space describes the liquid part of the market. Indeed, within the subspace $\operatorname{lin} S_{t}(\cdot, \omega)$, trading costs are linear so that marginal prices are independent of the size of the trade. The (RNA) condition means that, outside the liquid part of the market, it is possible to lower the trading costs without inducing arbitrage opportunities. When $S$ is linear, we have $\operatorname{lin} S_{t}=\mathbb{R}^{J}$ and the only cost process $\tilde{S} \leq S$ is $S$ itself, so the (RNA) condition reduces to the ordinary no-arbitrage condition. The following is thus a direct generalization of Example 9 .

Example 10 If $S$ satisfies the (RNA) condition and $D \equiv \mathbb{R}^{J}$, then $\mathcal{L}$ is linear and thus, $\mathcal{C}$ is closed.

Proof. If the linearity condition fails, there is an $x \in \mathcal{L}$ such that $S_{t}^{\infty}\left(-\Delta x_{t}\right)>$ 0 for some $t$ on a set $A \subset \mathcal{F}_{t}$ with $P(A)>0$. Such an $x$ is an arbitrage strategy for every cost process $\tilde{S}$ satisfying (4). Indeed, since $x \in \mathcal{L}$, the first condition in (4) implies that $x$ is self-financing for $\tilde{S}$. Moreover, if $\Delta x_{t} \notin \operatorname{lin} S_{t}$, then $\tilde{S}_{t}\left(\Delta x_{t}\right)<S_{t}^{\infty}\left(\Delta x_{t}\right) \leq 0$ while if $\Delta x_{t} \in \operatorname{lin} S_{t}$, then $\tilde{S}_{t}\left(\Delta x_{t}\right) \leq S_{t}^{\infty}\left(\Delta x_{t}\right)=$ $-S_{t}^{\infty}\left(-\Delta x_{t}\right)$. In either case, $\tilde{S}_{t}\left(\Delta x_{t}\right)<0$ on $A$ so $x$ is an arbitrage strategy for $\tilde{S}$. Thus, $S$ cannot satisfy the (RNA)-condition.

The (RNA) condition resembles the robust no-arbitrage condition introduced by Schachermayer [42] for the currency market model of Kabanov [22]. Whereas, the latter is concerned with claims with physical delivery (portfoliovalued claims), our model and the (RNA) condition defined above is designed for claims with cash-delivery (cash-valued claims).

Neither Example 9 nor 10 allow for portfolio constraints. Kreher [25] gave conditions that together with the robust no-arbitrage condition yield a closedness result in the currency market model of Kabanov [22] (or more precisely, in the nonconical extension of Kabanov's model studied in [32]). Kreher's result can be replicated in the present market model as follows.

We will say that a market model $(S, D)$ satisfies the robust no-arbitrage condition if there is a cost process $\tilde{S}$ satisfying $(4)$ such that $\left(\tilde{S}, D^{\infty}\right)$ is arbitragefree. We will use the notation

$$
\text { const } S_{t}(\omega)=\left\{x \in \mathbb{R}^{J} \mid S_{t}^{\infty}(x, \omega) \leq 0, S_{t}^{\infty}(-x, \omega) \leq 0\right\}
$$

for the constancy space of $S_{t}(\cdot, \omega)$; see [34, page 69]. Example 10 generalizes as follows.

Example 11 Assume that $S$ satisfies the (RNA) condition and that

$$
x \in \mathcal{N}_{0}, x_{t} \in D_{t}^{\infty}, \Delta x_{t} \in \text { const } S_{t} \Longrightarrow-x_{t} \in D_{t}^{\infty} .
$$

Then $\mathcal{L}$ is linear and thus, $\mathcal{C}$ is closed.

Proof. Assume, for contradiction, that there is an $x \in \mathcal{L}$ such that $-x \notin \mathcal{L}$. Then, there is a $t$ and an $A \subset \mathcal{F}_{t}$ with $P(A)>0$ such that $S_{t}^{\infty}\left(-\Delta x_{t}\right)>0$ on 
$A$. Indeed, if we had $S_{t}^{\infty}\left(-\Delta x_{t}\right) \leq 0$ almost surely for every $t$, we would get $\Delta x_{t} \in$ const $S_{t}$, so the extra condition would give $-x_{t} \in D_{t}^{\infty}$, in contradiction to $-x \notin \mathcal{L}$. Just as in the proof of Example 10, we can now deduce that $S$ cannot satisfy the (RNA)-condition.

The second condition in Example 11 holds, in particular, when the cone $D_{t}^{\infty}(\omega)$ is pointed (contains no lines) for every $t$ and $\omega$. This certainly holds when $D_{t}^{\infty}(\omega) \subseteq \mathbb{R}_{+}^{J}$, which means that any portfolio with a strictly negative component becomes infeasible when scaled up by a large enough constant.

The above examples relate the linearity condition in Theorem 8 to the notion of arbitrage which has had a central role in mathematical finance. In nonlinear models, however, arbitrage is not that relevant when it comes to pricing and hedging or to financial risk management in general. The following example (originally given as [31, Theorem 6.3]) gives a simple condition which allows for arbitrage but is still safe to assume in many situations in practice.

Example 12 If $D_{t}^{\infty}(\omega) \cap\left\{x \in \mathbb{R}^{J} \mid S_{t}^{\infty}(x, \omega) \leq 0\right\}=\{0\}$ almost surely for every $t=0, \ldots, T$ then $\mathcal{L}=\{0\}$ and thus, $\mathcal{C}$ is closed.

Proof. Let $x \in \mathcal{L}$. If $x_{t-1}=0$ the condition implies $x_{t}=0$. Since we had defined $x_{-1}=0$ we get $x=0$, by induction.

The condition in Example 12 holds, in particular, if $D^{\infty} \subseteq \mathbb{R}_{+}^{J}$ and if there is a componentwise strictly positive process $s=\left(s_{t}\right)_{t=0}^{T}$ with

$$
S_{t}(x, \omega) \geq s_{t}(\omega) \cdot x \quad \forall x \in \mathbb{R}^{J} .
$$

The latter condition can be written in terms of the subdifferential of $S_{t}(\cdot, \omega)$ at the origin as $s_{t}(\omega) \in \partial S_{t}(0, \omega)$; see Section 3. In limit order markets, the existence of a componentwise strictly positive $s_{t}(\omega) \in \partial S_{t}(0, \omega)$ means that the ask-prices of traded securities are always strictly positive; see [26] for a closer study of limit order markets.

\section{Fundamental theorem of asset pricing}

The classical result of Dalang, Morton and Willinger [4] states that an unconstrained linear model in finite discrete-time satisfies the no-arbitrage condition if and only if there exists an equivalent martingale measure, i.e. a probability measure equivalent to $P$ under which market prices are martingales. This result has been extended in various ways to allow e.g. for constraints [28, 10, 38] and claims with physical delivery $[42,23,32]$. Theorem 14 below gives a version for an unconstrained nonlinear market model when arbitrage is defined with respect to claims with cash-delivery as in Section 4.

In our model, the relevant dual elements are defined in terms of the conjugate

$$
S_{t}^{*}(v, \omega)=\sup _{x \in \mathbb{R}^{J}}\left\{x \cdot v-S_{t}(x, \omega)\right\}
$$


of the cost process. The classical conjugacy property of normal integrands (see $\left[33\right.$, Lemma 5]) implies that the conjugate of a cost process is also an $\left(\mathcal{F}_{t}\right)_{t=0^{-}}^{T}$ adapted sequence of convex normal integrands. Since $S_{t}(0, \omega)=0$, we have $S_{t}^{*}(\cdot, \omega) \geq 0$. When $S_{t}(\cdot, \omega)$ is sublinear, its conjugate takes the form of an indicator function. In the linear case $S_{t}(x, \omega)=s_{t}(\omega) \cdot x$, the conjugate is simply the indicator of $\left\{s_{t}(\omega)\right\}$. When $S_{t}(x, \omega)=\sup \left\{s \cdot x \mid s^{j} \in\left[\underline{s}_{t}^{j}(\omega), \bar{s}_{t}^{j}(\omega)\right]\right\}$, the conjugate is the indicator of the box $\left[\underline{s}_{t}(\omega), \bar{s}_{t}(\omega)\right]$.

Definition 13 A strictly positive adapted process y is said to be a strictly consistent deflator for a cost process $S$ if there is a price process $s$ with $s_{t} \in$ ridom $S_{t}^{*}$ almost surely for all $t$ such that ys is a martingale.

The term "strictly consistent deflator" deliberately resembles the term "strictly consistent price system" introduced in [42] in the context of the currency market model of Kabanov [22]. While a price system is a vector-valued process representing values of portfolios of assets, a deflator refers to a scalar-valued process describing the value of cash. In the linear case $S_{t}(x, \omega)=s_{t}(\omega) \cdot x$, strictly consistent deflators are the strictly positive adapted process $y$ such that $y s$ is a martingale. In this case, ys would be the strictly consistent price system in the sense of [42] for the conical model $\hat{K}_{t}(\omega):=\left\{x \in \mathbb{R}^{J} \mid s_{t}(\omega) \cdot x \geq 0\right\}$. If one of the components of $s$ is constant (see Examples 1 and 6 ), then strictly consistent deflators are of the form $y_{t}=\alpha E_{t} y_{T}$, where $\alpha$ is a positive scalar and $y_{T}$ is the density of an equivalent martingale measure.

The following is a nonlinear generalization of the fundamental theorem of asset pricing for linear cost processes given in Dalang, Morton and Willinger [4].

Theorem 14 If $S^{\infty}$ is finite-valued then $S$ satisfies the robust no-arbitrage condition if and only if it admits a strictly consistent deflator. Moreover, the deflator can be then chosen essentially bounded.

Proof. Since $S^{\infty}$ is finite-valued, the measurable function

$$
\varphi(\omega)=\max _{t=0, \ldots, T} \sup _{|x| \leq 1} S_{t}^{\infty}(x, \omega)
$$

is finite and nonnegative. Thus, $d \tilde{P} / d P=e^{-\varphi} / E e^{-\varphi}$ defines a measure $\tilde{P}$ equivalent to $P$. Since arbitrage is invariant under equivalent changes of measure, $S$ satisfies the robust no-arbitrage condition also under $\tilde{P}$. Moreover, $S^{\infty}$ is integrable under $\tilde{P}$ since for every nonzero $x \in \mathbb{R}^{J}$

$$
E^{\tilde{P}}\left|S_{t}^{\infty}(x, \omega)\right|=|x| E^{\tilde{P}}\left|S_{t}^{\infty}(x /|x|, \omega)\right| \leq|x| E^{\tilde{P}} \varphi=\frac{|x|}{E e^{-\varphi}} E \varphi e^{-\varphi}<\infty .
$$

Let $\tilde{S}$ be a sublinear cost process in the definition of the (RNA) condition. Then

$$
\bar{S}:=\frac{1}{2} \tilde{S}+\frac{1}{2} S^{\infty}
$$


defines a sublinear cost process that also satisfies (4) with $\tilde{S}$. By Example 10, the set

$$
\overline{\mathcal{C}}:=\left\{c \in \mathcal{M} \mid \exists x \in \mathcal{N}_{0}: \bar{S}_{t}\left(\Delta x_{t}\right)+c_{t} \leq 0\right\}
$$

is closed. Since $\bar{S}$ is arbitrage-free, we also have $\overline{\mathcal{C}} \cap \mathcal{M}_{+}^{1}=\{0\}$, where $\mathcal{M}_{+}^{1}$ is the set of nonnegative $\tilde{P}$-integrable claims. By the Kreps-Yan theorem (see e.g. [21]), there is a $y \in \mathcal{M}^{\infty}$ such that

$$
\begin{aligned}
& E \sum_{t=0}^{T} c_{t} y_{t}>0 \quad \forall c \in \mathcal{M}_{+}^{1} \backslash\{0\} \\
& E \sum_{t=0}^{T} c_{t} y_{t} \leq 0 \quad \forall c \in \overline{\mathcal{C}}^{1} .
\end{aligned}
$$

The first condition means that $y$ is strictly positive while the second means that $\sigma_{\overline{\mathcal{C}}^{1}}(y) \leq 0$. Since $\bar{S}$ is integrable under $\tilde{P}$, we then have, by Lemma 4 , that there is a $\tilde{P}$-martingale $v \in \mathcal{N}^{1}$ such that $\left(y_{t} \bar{S}_{t}\right)^{*}\left(v_{t}\right) \leq 0$ almost surely for every $t$. Since $\bar{S}$ is sublinear, this means that

$$
v_{t} / y_{t} \in \operatorname{dom} \bar{S}_{t}^{*}
$$

which, in turn, by [34, Corollary 13.3.4(a)], implies

$$
\bar{S}_{t}(x, \omega) \geq x \cdot v_{t} / y_{t} \quad \forall x \in \mathbb{R}^{J} .
$$

It follows that

$$
\begin{array}{ll}
S_{t}(x, \omega) \geq x \cdot v_{t} / y_{t} & \forall x \in \mathbb{R}^{J}, \\
S_{t}(x, \omega)>x \cdot v_{t} / y_{t} & \forall x \notin \operatorname{lin} S_{t}(\cdot, \omega)
\end{array}
$$

and then $v_{t} / y_{t} \in \operatorname{ridom} S_{t}^{*}$, by [34, Corollary 13.3.4(b)]. This means that $y$ is a strictly consistent deflator for $S$ under $\tilde{P}$. Since $v$ is a martingale under $\tilde{P}$, the process $\left(\tilde{v}_{t}\right)_{t=0}^{T}$ defined by $\tilde{v}_{t}:=v_{t} E_{t}(d \tilde{P} / d P)$ is a martingale under $P$ and then $\tilde{y}_{t}:=y_{t} E_{t}(d \tilde{P} / d P)$ defines a strictly consistent deflator under $P$. Moreover, since $y$ and $d \tilde{P} / d P$ are bounded, $\tilde{y}$ is also bounded.

To prove the converse, let $y$ be a strictly consistent deflator for $S$ and let $s$ be as in Definition 13. By [34, Corollary 13.3.4(b)], $S$ satisfies $(4)$ with $\tilde{S}_{t}(x, \omega)=$ $s_{t}(\omega) \cdot x$. It suffices to show that $\tilde{S}$ satisfies the no-arbitrage condition. To this end let $c \in \mathcal{M}_{+}$be such that $s_{t} \cdot \Delta x_{t}+c_{t} \leq 0$ almost surely for some $x \in \mathcal{N}_{0}$. We get

$$
0 \leq \sum_{t=0}^{T} y_{t} c_{t} \leq-\sum_{t=0}^{T} y_{t} s_{t} \cdot \Delta x_{t}=\sum_{t=0}^{T} x_{t-1} \cdot \Delta\left(y_{t} s_{t}\right) .
$$

Since $y s$ is a martingale and $x$ is adapted, the process

$$
M_{s}:=\sum_{t=0}^{s} x_{t-1} \cdot \Delta\left(y_{t} s_{t}\right)
$$


is a local martingale by [16, Theorem 1]. Moreover, since $M_{T} \geq 0$ almost surely, $M$ is a martingale by [16, Theorem 2]. Since $M_{0}=0$ we get

$$
E \sum_{t=0}^{T} y_{t} c_{t}=0 .
$$

Since $y$ is strictly positive and $c \in \mathcal{M}_{+}$, we must have $c=0$. Thus, $\tilde{S}_{t}$ satisfies the no-arbitrage condition.

For a model with bid-ask-spreads, Theorem 14 gives the following.

Example 15 (Bid-ask spreads) An unconstrained market model with $S_{t}(x, \omega)=$ $\sup \left\{s \cdot x \mid s^{j} \in\left[\underline{s}_{t}^{j}(\omega), \bar{s}_{t}^{j}(\omega)\right]\right\}$ satisfies the (RNA) condition if and only if there is strictly positive $y \in \mathcal{M}^{\infty}$ and an adapted process s evolving in $\mathrm{ri}\left[\underline{s}_{t}, \bar{s}_{t}\right]$ such that ys is a martingale. This is analogous to [19, Theorem 3.2(i)], which concerns the no-free lunch condition in a continuous time model. When one of the assets has a nonzero constant unit price, the process y obtained above is a constant multiple of a strictly positive martingale density. When $\underline{s}=\bar{s}$, we thus recover the classical result of Dalang, Morton and Willinger [4].

\section{References}

[1] J.-P. Aubin. Mathematical methods of game and economic theory, volume 7 of Studies in Mathematics and its Applications. North-Holland Publishing Co., Amsterdam, 1979.

[2] U. Çetin, R. A. Jarrow, and P. Protter. Liquidity risk and arbitrage pricing theory. Finance Stoch., 8(3):311-341, 2004.

[3] J. Cvitanić and I. Karatzas. Convex duality in constrained portfolio optimization. Ann. Appl. Probab., 2(4):767-818, 1992.

[4] R. C. Dalang, A. Morton, and W. Willinger. Equivalent martingale measures and no-arbitrage in stochastic securities market models. Stochastics Stochastics Rep., 29(2):185-201, 1990.

[5] F. Delbaen and W. Schachermayer. The Mathematics of Arbitrage. Springer Finance. Springer-Verlag, Berlin Heidelberg, 2006.

[6] M. A. H. Dempster, I. V. Evstigneev, and M. I. Taksar. Asset pricing and hedging in financial markets with transaction costs: An approach based on the Von Neumann-Gale model. Annals of Finance, 2(4):327-355, 2006.

[7] J. C. Dermody and R. T. Rockafellar. Cash stream valuation in the face of transaction costs and taxes. Math. Finance, 1(1):31-54, 1991.

[8] J. C. Dermody and R. T. Rockafellar. Tax basis and nonlinearity in cash stream valuation. Math. Finance, 5(2):97-119, 1995. 
[9] I. Ekeland and R. Temam. Convex analysis and variational problems. North-Holland Publishing Co., Amsterdam, 1976. Translated from the French, Studies in Mathematics and its Applications, Vol. 1.

[10] I. V. Evstigneev, K. Schürger, and M. I. Taksar. On the fundamental theorem of asset pricing: random constraints and bang-bang no-arbitrage criteria. Math. Finance, 14(2):201-221, 2004.

[11] H. Föllmer. On the representation of semimartingales. Ann. Probability, 1:580-589, 1973 .

[12] H. Föllmer and A. Schied. Convex measures of risk and trading constraints. Finance Stoch., 6(4):429-447, 2002.

[13] H. Föllmer and A. Schied. Stochastic finance, volume 27 of de Gruyter Studies in Mathematics. Walter de Gruyter \& Co., Berlin, extended edition, 2004. An introduction in discrete time.

[14] M. B. Haugh and L. Kogan. Pricing american options: a duality approach. Oper. Res., 52(2):258-270, 2004.

[15] J. E. Ingersoll. Theory of Financial Decision Making. Studies in Financial Economics. Rowman \& Littlefield Publishers, Inc., Lanham, MD, 1987.

[16] J. Jacod and A. N. Shiryaev. Local martingales and the fundamental asset pricing theorems in the discrete-time case. Finance Stoch., 2(3):259-273, 1998.

[17] S. Jaschke and U. Küchler. Coherent risk measures and good-deal bounds. Finance Stoch., 5(2):181-200, 2001.

[18] E. Jouini and H. Kallal. Arbitrage in securities markets with short-sales constraints. Math. Finance, 5(3):197-232, 1995.

[19] E. Jouini and H. Kallal. Martingales and arbitrage in securities markets with transaction costs. J. Econom. Theory, 66(1):178-197, 1995.

[20] E. Jouini and C. Napp. Arbitrage and investment opportunities. Finance Stoch., 5(3):305-325, 2001.

[21] E. Jouini, C. Napp, and W. Schachermayer. Arbitrage and state price deflators in a general intertemporal framework. J. Math. Econom., 41(6):722$734,2005$.

[22] Y. M. Kabanov. Hedging and liquidation under transaction costs in currency markets. Finance and Stochastics, 3(2):237-248, 1999.

[23] Y. M. Kabanov and M. Safarian. Markets with transaction costs. Springer Finance. Springer-Verlag, Berlin, 2009. Mathematical theory. 
[24] K. Kaval and I. Molchanov. Link-save trading. J. Math. Economics, 42:710728, 2006.

[25] D. Kreher. Hedging of portfolio-valued claims under convex transaction costs and portfolio constraints. Master's thesis, Humboldt-Universität zu Berlin, 2009.

[26] P. Malo and T. Pennanen. Reduced form modeling of limit order markets. Quantitative Finance, to appear.

[27] A. Mas-Collel, M.D. Whinston, and J.R. Green. Microeconomic Theory. Oxford University Press, New York, 1995.

[28] C. Napp. The Dalang-Morton-Willinger theorem under cone constraints. $J$. Math. Econom., 39(1-2):111-126, 2003. Special issue on equilibrium with asymmetric information.

[29] T. Pennanen. Arbitrage and deflators in illiquid markets. Finance and Stochastics, 15(1):57-83, 2011.

[30] T. Pennanen. Convex duality in stochastic optimization and mathematical finance. Mathematics of Operations Research, 36(2):340-362, 2011.

[31] T. Pennanen. Superhedging in illiquid markets. Mathematical Finance, 21(3):519-540, 2011.

[32] T. Pennanen and I. Penner. Hedging of claims with physical delivery under convex transaction costs. SIAM Journal on Financial Mathematics, 1:158178,2010 .

[33] R. T. Rockafellar. Integrals which are convex functionals. Pacific J. Math., 24:525-539, 1968.

[34] R. T. Rockafellar. Convex analysis. Princeton Mathematical Series, No. 28. Princeton University Press, Princeton, N.J., 1970.

[35] R. T. Rockafellar. Conjugate duality and optimization. Society for Industrial and Applied Mathematics, Philadelphia, Pa., 1974.

[36] R. T. Rockafellar and R. J.-B. Wets. Variational analysis, volume 317 of Grundlehren der Mathematischen Wissenschaften [Fundamental Principles of Mathematical Sciences]. Springer-Verlag, Berlin, 1998.

[37] L. C. G. Rogers. Monte Carlo valuation of American options. Math. Finance, 12(3):271-286, 2002.

[38] D. B. Rokhlin. An extended version of the Dalang-Morton-Willinger theorem under convex portfolio constraints. Theory Probab. Appl., 49(3):429443, 2005. 
[39] D. B. Rokhlin. The Kreps-Yan theorem for $L^{\infty}$. Int. J. Math. Math. Sci., (17):2749-2756, 2005.

[40] D. B. Rokhlin. Martingale selection problem and asset pricing in finite discrete time. Electron. Comm. Probab., 12:1-8, 2007.

[41] W. Schachermayer. A Hilbert space proof of the fundamental theorem of asset pricing in finite discrete time. Insurance Math. Econom., 11(4):249$257,1992$.

[42] W. Schachermayer. The fundamental theorem of asset pricing under proportional transaction costs in finite discrete time. Math. Finance, 14(1):1948, 2004 . 